\title{
Does rapid genetic counseling and testing in newly diagnosed breast cancer patients cause additional psychosocial distress? Results from a randomized clinical trial
}

\author{
Marijke R. Wevers, MD ${ }^{1,2}$, Margreet G.E.M. Ausems, MD, PhD², \\ Senno Verhoef, MD, PhD², Eveline M.A. Bleiker, PhD ${ }^{1}$, Daniela E.E. Hahn, MSc ${ }^{4}$, \\ Titia Brouwer' ${ }^{2}$, Frans B.L. Hogervorst, PhD ${ }^{3}$, Rob B. van der Luijt, PhD², \\ Thijs van Dalen, MD, $\mathrm{PhD}^{5}$, Evert $\mathrm{B}$. Theunissen, $\mathrm{MD}^{6}$, \\ Bart van Ooijen, MD, $\mathrm{PhD}^{7}$, Marnix A. de Roos, MD, $\mathrm{PhD}^{8}$, \\ Paul J. Borgstein, MD, PhD ${ }^{9}$, Bart C. Vrouenraets, MD, $\mathrm{PhD}^{10}$, \\ Eline Vriens, MD, PhD ${ }^{11}$, Wim H. Bouma, MD, PhD ${ }^{12}$, Herman Rijna, MD, PhD ${ }^{13}$, \\ Johannes P. Vente, MD, PhD ${ }^{14}$, Jacobien M. Kieffer, $\mathrm{PhD}^{1}$, Heiddis B. Valdimarsdottir, $\mathrm{PhD}^{15}$, \\ Emiel J.Th. Rutgers, MD, PhD ${ }^{16}$, Arjen J. Witkamp, MD, PhD ${ }^{17}$ and Neil K. Aaronson, PhD ${ }^{1}$
}

\begin{abstract}
Purpose: Female breast cancer patients carrying a BRCA1/2 mutation have an increased risk of second primary breast cancer. Rapid genetic counseling and testing (RGCT) before surgery may influence choice of primary surgical treatment. In this article, we report on the psychosocial impact of RGCT.

Methods: Newly diagnosed breast cancer patients at risk for carrying a $B R C A 1 / 2$ mutation were randomized to an intervention group (offer of RGCT) or a usual care control group (ratio 2:1). Psychosocial impact and quality of life were assessed with the Impact of Events Scale, Hospital Anxiety and Depression Scale, Cancer Worry Scale, and the EORTC QLQ-C30 and QLQ-BR23. Assessments took place at study entry and at 6- and 12-month follow-up visits.
\end{abstract}

Results: Between 2008 and 2010, 265 patients were recruited into the study. Completeness of follow-up data was more than $90 \%$. Of the 178 women in the intervention group, 177 had genetic counseling, of whom 71 (40\%) had rapid DNA testing and 59 (33\%) received test results before surgery. Intention-to-treat and per-protocol analyses showed no statistically significant differences between groups over time in any of the psychosocial outcomes.

Conclusions: In this study, RGCT in newly diagnosed breast cancer patients did not have any measurable adverse psychosocial effects.

Genet Med advance online publication 23 April 2015

Key Words: breast cancer; BRCA1/2; genetic counseling and testing; psychosocial distress; quality of life

\section{INTRODUCTION}

Female breast cancer patients who carry a BRCA1 or BRCA2 gene mutation have an increased risk of $20-55 \%$ for developing a second primary breast cancer ${ }^{1}$ and are at increased risk for ovarian cancer. The risk of contralateral breast cancer is highest for carriers who were diagnosed with their first breast cancer at a young age. ${ }^{1}$ Between 18 and $29 \%$ of carriers with a favorable prognosis opt for immediate or, more often, delayed contralateral prophylactic mastectomy $(\mathrm{CPM})$ to reduce their risk of a second breast cancer. ${ }^{2-4}$ Several studies have shown that a CPM leads to a large reduction (up to 95\%) in the risk of contralateral breast cancer, with increasing evidence of improved breast cancer-specific survival. ${ }^{5,6}$

Advances in the technology and logistics of genetic testing of the BRCA1 and BRCA2 genes have made it possible to offer high-risk breast cancer patients the opportunity to undergo rapid genetic counseling and testing (RGCT) during the time period between diagnosis and primary surgery. Women who

\footnotetext{
${ }^{1}$ Division of Psychosocial Research and Epidemiology, The Netherlands Cancer Institute, Amsterdam, The Netherlands; ${ }^{2}$ Division of Biomedical Genetics, University Medical Center Utrecht, Utrecht, The Netherlands; ${ }^{3}$ Family Cancer Clinic, The Netherlands Cancer Institute, Amsterdam, The Netherlands; ${ }^{4}$ Department of Psychosocial Counseling, The Netherlands Cancer Institute, Amsterdam, The Netherlands; ${ }^{5}$ Division of Surgery, Diakonessen Hospital, Utrecht, The Netherlands; ${ }^{6}$ Division of Surgery, St. Antonius Hospital, Nieuwegein, The Netherlands; ${ }^{7}$ Division of Surgery, Meander Medical Center, Amersfoort, The Netherlands; ${ }^{8}$ Division of Surgery, Rivierenland Hospital, Tiel, The Netherlands; ${ }^{9}$ Division of Surgery, Onze Lieve Vrouwe Gasthuis, Amsterdam, The Netherlands; ${ }^{10}$ Division of Surgery, St. Lucas Andreas Hospital, Amsterdam, The Netherlands; ${ }^{11}$ Division of Surgery, Tergooi Hospitals, Blaricum, The Netherlands; ${ }^{12}$ Division of Surgery, Gelre Hospitals, Apeldoorn, The Netherlands; ${ }^{13}$ Division of Surgery, Kennemer Gasthuis, Haarlem, The Netherlands; ${ }^{14}$ Division of Surgery, Zuwe Hofpoort Hospital, Woerden, The Netherlands; ${ }^{15}$ Department of Psychology, Reykjavik University, Reykjavik, Iceland; ${ }^{16}$ Division of Surgery, The Netherlands Cancer Institute, Amsterdam, The Netherlands; ${ }^{17}$ Division of Surgery, University Medical Center Utrecht, Utrecht, The Netherlands. Correspondence: Neil K. Aaronson (n.aaronson@nki.nl)
} 
receive timely information regarding their carrier status may incorporate this knowledge into decisions regarding their primary treatment, including the possibility of an immediate CPM.

Several studies have evaluated long-term satisfaction and psychosocial outcomes of risk-reducing surgery in breast cancer patients with a positive family history of the disease. ${ }^{7-9}$ Overall, most women reported high levels of satisfaction, ${ }^{8,9}$ favorable effects on emotional stability and stress, ${ }^{8}$ and significantly lower levels of breast cancer concerns than women who had not undergone CPM. ${ }^{9}$ Distress levels and quality of life were comparable with those of women who did not undergo CPM. ${ }^{9}$ Negative effects were reported for body appearance, sense of femininity, ${ }^{8}$ and sexuality. ${ }^{7}$ However, these were all retrospective studies focusing on a delayed CPM in patients not aware of their genetic status at the time of their breast cancer diagnosis ${ }^{8,9}$ or describing a group of both affected and unaffected women. ${ }^{7}$

Less is known about the psychosocial impact of RGCT and of immediate CPM at the time of the initial breast cancer diagnosis. In a retrospective study of breast cancer patients who underwent RGCT, approximately one-quarter of women reported clinically relevant levels of breast cancer-specific distress at a mean follow-up of 29 months. However, their distress levels were comparable with those of early stage breast cancer patients treated with radiotherapy, on average, 21 months earlier. ${ }^{10}$ This suggests that the observed distress was probably not due to having undergone RGCT. In a prospective, observational study, Tercyak et al. ${ }^{4}$ found no differences in quality of life or distress levels between women with breast cancer who had undergone RGCT and chose immediate CPM $(n=29)$ versus those who chose unilateral mastectomy or breast-conserving surgery $(n=118)$. This suggests that undergoing an immediate CPM does not cause additional distress. However, to date, no randomized controlled studies have been conducted regarding the psychosocial impact of RGCT. Some concern continues to be voiced that offering genetic counseling and testing to a woman who has just received a breast cancer diagnosis may be "too much, too soon."11

We have performed a multicenter, randomized, clinical trial of the behavioral and psychosocial impact of RGCT in newly diagnosed breast cancer patients with a high risk of having a hereditary form of the disease. We recently reported the results of the behavioral outcomes from this trial..$^{12}$ Briefly, we found that when RGCT is offered routinely to high-risk breast cancer patients, almost all women accept rapid genetic counseling and approximately $40 \%$ opt for a rapid DNA test of the BRCA1/2 genes. Based on an intention-to-treat (ITT) analysis, RGCT did not have a significant impact on choice of primary surgery. However, a per-protocol analysis indicated that women who received their DNA test results before primary surgery opted significantly more often for an immediate CPM than women receiving usual care (UC).

In the current article, we report on the psychosocial outcomes from this trial. Specifically, we investigated the impact of RGCT on: (i) cancer-related worry and distress; (ii) health-related quality of life (HRQL); (iii) body image and sexuality; (iv) satisfaction with decision making; and (v) perceived risk of having hereditary breast cancer. Before starting the trial, we hypothesized that, in comparison with the UC group, women in the RGCT group would opt significantly more often for an immediate CPM and therefore would report significantly lower levels of cancer worries and cancer-related distress at follow-up. We also hypothesized that women in the RGCT group would have higher levels of decisional satisfaction. Conversely, following our hypothesis of an increased uptake of immediate CPM in the RGCT group, we anticipated that women in the RGCT group would report significantly more problems with body image and sexuality than those in the UC group. Hence, cancer worries, cancer-related distress, decisional satisfaction, body image, and sexuality were considered primary outcomes. We did not expect any significant differences between the RGCT and the UC groups on other HRQL outcomes, which were therefore considered secondary outcomes. ${ }^{13}$

\section{Study population}

\section{MATERIALS AND METHODS}

Trial participants were newly diagnosed female breast cancer patients with at least a $10 \%$ risk of carrying a $B R C A 1$ or $B R C A 2$ gene mutation according to Dutch guidelines. ${ }^{14}$ Patients with invasive breast cancer and ductal carcinoma in situ could participate. Exclusion criteria were already having undergone surgical treatment for this breast cancer, age younger than 18 years, not being able to read or write in Dutch, and already having undergone genetic counseling and testing. We included patients with unilateral and bilateral breast cancer because a bilateral mastectomy is not always necessary in the case of bilateral breast cancer. ${ }^{12}$

\section{Study design}

Details of the design and methods of this prospective, randomized, controlled trial are reported elsewhere. ${ }^{12,13}$ Briefly, between November 2008 and December 2010, eligible patients were recruited from 12 hospitals in the Netherlands. The study was approved by the medical ethics committees of all participating hospitals, and all patients provided informed consent before randomization. Two-thirds of the participants were allocated to the intervention group that was routinely invited to undergo RGCT, and one-third of participants were allocated to a UC control group. Randomization was stratified by hospital. ${ }^{12,13}$ Given the nature of the intervention, blinding of the participants was not possible. The RGCT group was offered an appointment with a clinical geneticist within 5 working days after recruitment and the opportunity to undergo rapid DNA testing, with test results provided within approximately 4 weeks. Women in the UC group could be referred to a clinical geneticist by their treating physician. Although referral to RGCT was also possible for women in the UC group, this most typically involved GCT after completion of primary treatment.

\section{Data collection}

All participants were asked to complete questionnaires at baseline (prior to randomization) and at 6- and 12-month follow-up 
Table 1 Psychosocial measures

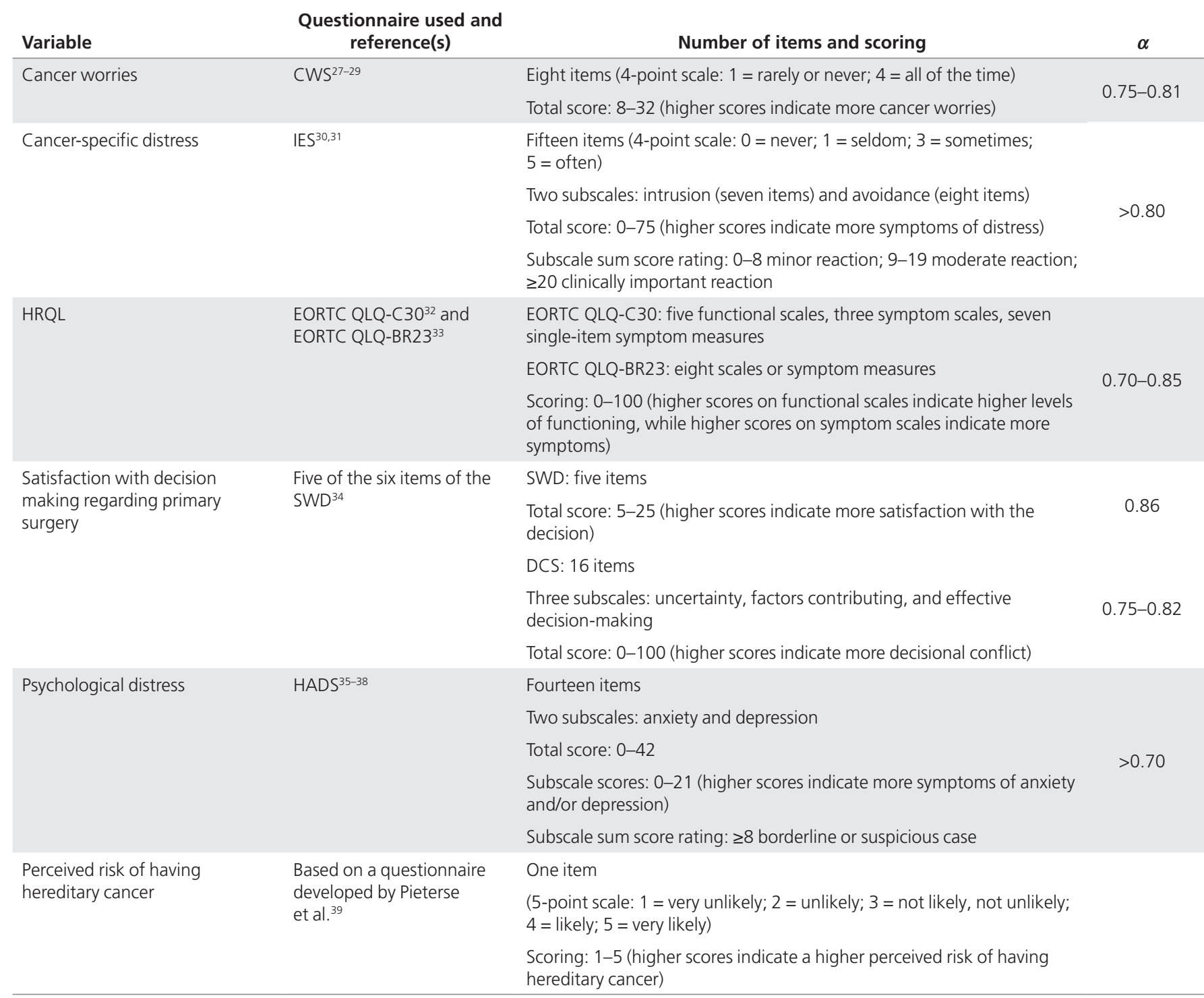

CWS, Cancer Worry Scale; DCS, Decisional Conflict Scale; EORTC QLQ-BR23, European Organization for Research and Treatment of Cancer Breast Cancer scale; EORTC QLQC30, European Organization for Research and Treatment of Cancer HRQL; HADS, Hospital Anxiety and Depression Scale; HRQL, health-related quality of life; IES, Impact of Events Scale; n.a., not applicable; SWD, Satisfaction with Decision Scale.

visits. ${ }^{13}$ The questionnaire assessed sociodemographics, cancer worries, cancer-specific distress, perceived risk of having hereditary breast cancer, HRQL, and satisfaction with choice of primary surgery. Details of the specific scales used to assess these psychosocial outcomes are described in Table 1.

Clinical data were abstracted from the medical records and included breast cancer diagnosis (unilateral or bilateral, TNM stage), DNA test results, hospital, cancer history, type of breast cancer therapy, and type of prophylactic surgery.

\section{Statistical analyses}

Descriptive statistics were used to characterize the study sample. We used repeated measures mixed effect models to evaluate short- and long-term group differences over time in psychosocial outcomes. The models included random effects for the intercept and the stratification variable (hospital) to account for homogeneity between subjects within strata. Specific contrasts were modeled between groups and follow-up assessment using a maximum likelihood solution. These analyses were conducted both on an ITT and per-protocol basis. For the primary ITT analyses, all women in the RGCT group were included, regardless of whether they actually received RGCT. In the per-protocol analyses, women in the intervention group who underwent DNA testing and received their test results before first surgery were compared with all women allocated to the control group. Additionally, we modeled changes in psychosocial outcome from baseline to 12-month follow-up for the total study sample.

The sample size calculations were based primarily on the uptake of bilateral mastectomy. With a sample size ratio of 2:1 (RGCT:UC), power set at 0.80 , and $\alpha$ set at $0.05,170$ patients 
were required in the RGCT group and 85 were required in the UC group to detect a difference of 18 vs. $5 \%$ in the uptake of bilateral mastectomy. For the psychosocial outcomes, a total sample size of 255 women would be adequate to detect a 0.5 SD between group difference in mean scores (a 0.50 effect size; minimum required $n=67$ cases per group).

Short- and long-term differences in mean change scores were accompanied by effect sizes and their confidence intervals (CIs). Effects sizes were expressed as Cohen's $d$ statistic and were calculated by dividing the difference in the mean change scores between the RGCT group and the UC group by the pooled baseline SD. Effect sizes of $(-) 0.2$ were considered small, $(-) 0.5$ were considered moderate, and $(-) 0.8$ were considered large. ${ }^{15}$ Effect sizes of approximately (-) 0.5 are considered to be clinically relevant. ${ }^{15}$ Effects for primary outcomes were considered significant at $P<0.05$, and effects for secondary outcomes were considered significant at $P<0.01$. The latter, more conservative significance level was introduced to reduce any possible effect resulting from multiple testing.

The trial is registered at www.clinicaltrials.gov, NCT00783822. Full details of the trial protocol can be found there.

\section{RESULTS}

Between November 2008 and December 2010, we invited 352 women to participate in the study. Eighty women declined and one withdrew consent at follow-up. Additionally, six women who were randomly assigned were subsequently determined not to have met inclusion criteria. In total, 265 women were included in the study, of whom 178 were allocated to the RGCT group and 87 to the UC group.

Study participants were younger than nonparticipants (mean age of 44.9 vs. 53.0 years, $P<0.001$ ). The most commonly

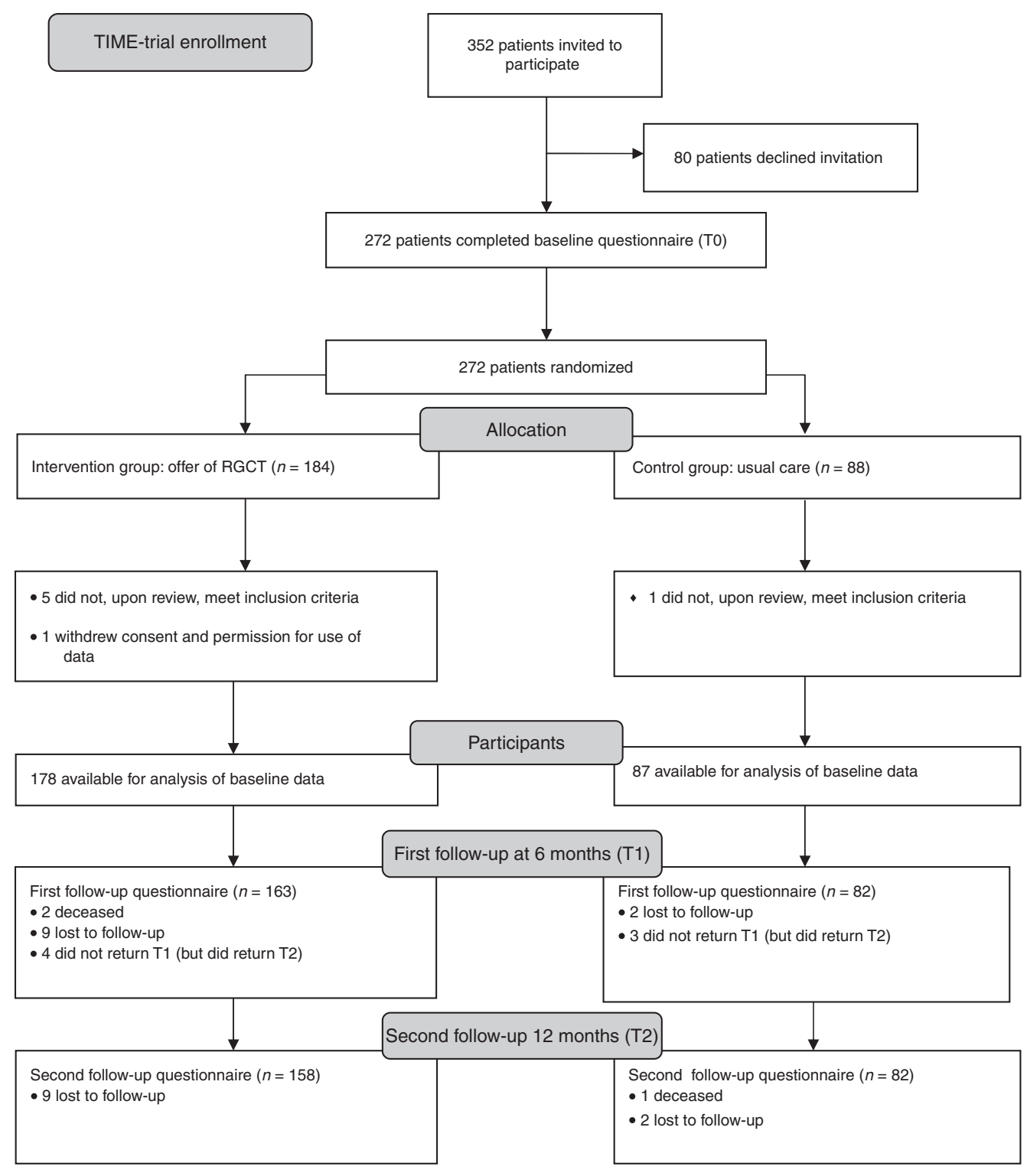

Figure 1 CONSORT (Consolidated Standards of Reporting Trials) diagram. 
Table 2 Characteristics of study participants

\begin{tabular}{|c|c|c|}
\hline & $\begin{array}{c}\text { Intervention } \\
\text { group (RGCT), } \\
\quad N=178\end{array}$ & $\begin{array}{c}\text { Control } \\
\text { group (UC), } \\
N=87\end{array}$ \\
\hline Age at diagnosis (years) & $\begin{array}{c}\text { Mean: } \\
44.9(11.4 \text { SD) } \\
\text { range: } 19-79\end{array}$ & $\begin{array}{c}\text { Mean: } \\
44.8(11.2 \text { SD), } \\
\text { range: } 25-72\end{array}$ \\
\hline \multicolumn{3}{|l|}{ Marital status } \\
\hline Single/widow/divorced & $36(20.2 \%)$ & $13(14.9 \%)$ \\
\hline Partner/married & $142(79.8 \%)$ & $74(85.1 \%)$ \\
\hline \multicolumn{3}{|l|}{ Patient's country of birth } \\
\hline Netherlands & $162(91 \%)$ & $77(88.5 \%)$ \\
\hline Other & $16(9 \%)$ & $10(11.5 \%)$ \\
\hline \multicolumn{3}{|l|}{ Occupational status } \\
\hline Employed & $128(71.9 \%)$ & $72(82.8 \%)$ \\
\hline $\begin{array}{l}\text { Not employed (including } \\
\text { retired, student, volunteer, and } \\
\text { homemaker) }\end{array}$ & $50(28.1 \%)$ & $15(17.2 \%)$ \\
\hline Children (yes) & $130(73 \%)$ & $64(74 \%)$ \\
\hline \multicolumn{3}{|l|}{ Education (n) } \\
\hline Primary/middle school & $41(23.0 \%)$ & $22(25.3 \%)$ \\
\hline High school & $42(23.6 \%)$ & $27(31.0 \%)$ \\
\hline College/university & $92(51.7 \%)$ & $38(43.7 \%)$ \\
\hline Other & $3(1.7 \%)$ & $0(0 \%)$ \\
\hline \multicolumn{3}{|l|}{ Laterality of current breast cancer } \\
\hline Unilateral & $173(97.2 \%)$ & $79(90.8 \%)$ \\
\hline Bilateral & $5(2.8 \%)$ & $8(9.2 \%)$ \\
\hline \multicolumn{3}{|l|}{ History of breast cancer } \\
\hline |psilateral & $2(1.1 \%)$ & $0(0 \%)$ \\
\hline Contralateral & $12(6.7 \%)$ & $9(10.3 \%)$ \\
\hline None & $164(92.1 \%)$ & $78(89.7 \%)$ \\
\hline \multicolumn{3}{|l|}{ Tumor stage } \\
\hline Stage 0 (ductal carcinoma in situ) & $10(5.6 \%)$ & $7(8.0 \%)$ \\
\hline Stage 1 & $65(36.5 \%)$ & $35(40.2 \%)$ \\
\hline Stage 2 & $78(43.8 \%)$ & $36(41.4 \%)$ \\
\hline Stage 3 & $20(11.2 \%)$ & $8(9.2 \%)$ \\
\hline Missing & $5(2.8 \%)$ & $1(1.1 \%)$ \\
\hline \multicolumn{3}{|l|}{ Adjuvant treatment } \\
\hline Chemotherapy (yes) & $114(64.0 \%)$ & $58(66.7 \%)$ \\
\hline Herceptin (yes) & $26(14.6 \%)$ & $10(11.5 \%)$ \\
\hline Hormonal treatment (yes) & $97(54.5 \%)$ & $47(54.0 \%)$ \\
\hline
\end{tabular}

$N$, number; RGCT, rapid genetic counseling and testing; SD, standard deviation; UC, usual care.

reported reasons for declining participation were not seeing the value of RGCT, perceiving participation in the trial as being too burdensome (reported by approximately one-quarter of nonparticipants), preferring to first undergo surgery before undergoing GCT, not wanting to complete questionnaires, and having close relatives who do not want to know whether hereditary breast cancer runs in the family.
At 6-month follow-up, 245 (92\%) questionnaires were returned; at 12-month follow-up, 240 (91\%). Further details of the study flow are described in the CONSORT (Consolidated Standards of Reporting Trials) diagram (Figure 1).

There were no statistically significant differences observed between the RGCT and UC groups on sociodemographic or clinical background variables except for bilaterality of the breast cancer (Table 2). Significantly more women in the UC group were diagnosed with bilateral synchronous breast cancer than in the RGCT group (9.2 vs. $2.8 \%, P=0.02$ ). For all subsequent group comparisons, we performed sensitivity analyses, adjusting statistically for a diagnosis of bilateral breast cancer. The results of these sensitivity analyses did not differ from those of the primary analyses, and thus only the latter results are reported here.

All but one woman in the RGCT group underwent genetic counseling within approximately 1 year of diagnosis, with a median time between randomization and the first genetic counseling consultation of 4 days (range $=0-374$ days). One hundred seventy-one women in the RGCT group (96\%) underwent genetic testing, of whom $59(33 \%)$ received their test results before their first surgery. Women reported various reasons for not undergoing DNA testing. Some women wanted more time to consider this option. Others had relatives who had previously undergone testing without finding a mutation, and thus felt less compelled to do so themselves. Still other women chose not to undergo testing because, although they themselves had developed breast cancer at a relatively young age (fulfilling criteria for testing eligibility), they did not have any relatives affected with breast or ovarian cancer.

In the UC group, 62 women (71\%) underwent genetic counseling after their breast cancer diagnosis, of whom 54 (62\%) had DNA testing. The cumulative numbers of women in the UC group who received DNA test results during follow-up were 9 (10\%) prior to undergoing surgery, $21(24 \%)$ within half a year after randomization, and 41 (47\%) within 1 year after randomization. The remaining 13 women who underwent DNA testing received their results subsequent to the 12 -month follow-up period.

In total, 22 pathogenic $B R C A 1 / 2$ mutations were found (17 in the RGCT group and 5 in the UC group, $P=0.88$ ). Overall, this represents $9.8 \%$ of those tested, which reflected our expectations. Further details are described elsewhere. ${ }^{12}$

\section{ITT analyses}

Results of the ITT analyses based on overall model effects indicated no statistically significant short- or long-term differences between the RGCT and the UC group in cancer worries, cancer specific distress, anxiety and depression, (breast) cancer-specific HRQL, satisfaction with decision making, or decisional conflict (Supplementary Table S1 online). The only statistically significant group difference over time was in the perceived risk of having hereditary breast cancer, with a greater decline from baseline to 12-month followup in the RGCT as compared with the UC group $(P=0.005$, 
effect size (ES) $=-0.32$; Supplementary Table S1 online). Effect sizes for the primary outcomes were small, ranging from 0.00 for the Impact of Events Scale (IES) intrusion scale to -0.26 for the sexual functioning scale at 6-month follow-up, and from -0.04 for the IES total score to -0.16 for the sexual functioning scale at 12 -month follow-up. Effect sizes for 4 of the 24 secondary outcomes (future perspective, breast symptoms, dyspnea, and perceived risk of having hereditary cancer) were more than 0.3 at 6- and/or 12-month follow-up, all in favor of the RGCT group.

\section{Per-protocol analyses}

For the per-protocol analyses, women in the intervention group ( $n=59$ at baseline, and $n=56$ at 6- and 12-month follow-up) who received their DNA test results before primary surgery were compared with the intact control group $(n=87$ at baseline and $n=82$ at 6 - and 12-month follow-up). Again, no statistically significant group differences over time were observed for any of the psychosocial outcomes, with the exception of the perceived risk of having hereditary breast cancer $(P=0.008$, $\mathrm{ES}=-0.36$ ). The latter results were similar to those reported for the ITT analysis (See Supplementary Table S2 online).

\section{Course of psychosocial measures during follow-up}

When analyzing the study sample as a whole (i.e., the RGCT and the UC groups combined), we observed a significant change over time in almost all psychosocial measures, with $P<0.001$ except for cognitive function $(P=0.02)$, insomnia $(P=0.01)$, and diarrhea $(P=0.91)$ (not shown in tabular form). More specifically, participants reported, on average, significantly fewer cancer worries and less cancer-specific and general psychological distress at 6-month follow-up as compared with baseline, with a generally stable pattern observed between 6- and 12-month follow-up. By contrast, self-reported fatigue, physical, role, cognitive and social functioning, global HRQL, body image, and sexuality declined significantly from baseline to 6-month follow-up. For most of these outcomes, there was a return to (near) baseline values at 12-month follow-up. This recovery at 12-month follow-up was less pronounced for body image and sexuality.

\section{DISCUSSION}

To the best of our knowledge, this is the first randomized controlled trial to publish data on the psychosocial impact of RGCT in newly diagnosed high-risk breast cancer patients. Overall, the women in our study reported substantial distress soon after diagnosis, especially anxiety and intrusive thoughts, which decreased over time. Such heightened levels of distress and compromised HRQL have also been described in breast cancer patients without a high-risk profile. ${ }^{16-18}$ Unfortunately, we did not have data available for breast cancer patients without a high-risk profile for comparison of distress levels.

Contrary to our original expectations, at follow-up, women in the RGCT group did not report significantly lower levels of cancer worries or cancer-related distress or more problems with body image or sexuality than the UC group. However, these hypotheses were based on the expectation of a significantly higher uptake of immediate CPM in the RGCT group. As reported in our previous work, this expectation was not met, based on ITT analyses. ${ }^{12}$

On the basis of the per-protocol analysis of our clinical data, significant group differences were observed in the uptake of CPM. That is, women who received their DNA test results before surgery (59/178 women in the RGCT group) opted significantly more often for direct bilateral mastectomy (BLM) than patients who received UC (22 vs. 9.2\%; OR 3.09, CI 1.158.31, $P=0.03)$. Nevertheless, the per-protocol analysis of the psychosocial outcomes did not show any significant group differences in cancer worries or distress, HRQL, body image, or sexuality at follow-up.

In the ITT and per-protocol analyses, effect sizes for all primary outcomes and most secondary outcomes were small. For a few secondary outcomes, the effect sizes were somewhat larger, with all differences favoring the RGCT group.

Combined, these results provide preliminary evidence to suggest that undergoing RGCT and opting for an immediate CPM does not carry with it any significant psychosocial effects, either positive or negative. The observed decline over time in distress levels in both groups likely reflects the passage of time, as described by Schlich-Bakker et al. ${ }^{19}$

Many professionals in the field of breast cancer care, including both surgeons and psychosocial care professionals, have been reluctant to discuss breast cancer genetics and its implications with women who may already be distressed because of just having received a cancer diagnosis. ${ }^{11,20}$ Professional organizations have recommended limiting RGCT to exceptional situations until research into its behavioral and psychosocial impact becomes available. ${ }^{21,22}$ By contrast, qualitative data indicate that women with breast cancer have a positive attitude toward RGCT, ${ }^{23}$ and results of a pilot study have suggested that genetic counseling before definitive surgery does not increase distress levels. ${ }^{24}$ The results of our trial provide additional evidence in this regard. However, we emphasize the need to confirm our results in future studies.

We did not have any specific hypothesis with regard to the impact of RGCT on the perceived risk of having hereditary breast cancer. However, because the majority of women in the RGCT group were expected to receive counseling and testing, and because approximately $90 \%$ of them were expected not to carry a pathogenic $B R C A 1 / 2$ mutation, lower levels of perceived risk at follow-up might be expected. ${ }^{25}$ We observed a greater decline in perceived risk of having hereditary breast cancer in the RGCT group than the UC group. This suggests that RGCT may have induced a more realistic view of the chance of having hereditary breast cancer. However, given the cumulative number of women in the UC group who had counseling and received their test results within the 6-and 12-month follow-up periods ( 24 and $47 \%$, respectively), it is difficult to explain why their level of perceived risk remained relatively stable over time (rather than decreasing as in the RGCT group, albeit more slowly). We would note that the decrease in the perceived risk 
of having hereditary breast cancer observed in the RGCT group did not translate into concomitant reductions in cancer worries. Further research is needed to better understand the factors related to changes in risk perception over time.

Our study had several limitations that should be noted. First, we did not have complete information regarding the number of women who received psychosocial counseling during the course of the study. We know that women in the RGCT group were routinely offered referral to such counseling in the genetics departments involved, and we know how many made use of these services. However, we do not know how many women made use of psychosocial services outside of this setting.

Second, participants in our study were relatively highly educated compared with the general population, and they were significantly younger than patients who declined participation. Also, approximately one-quarter of nonparticipants considered participating in the trial as too burdensome, although we do not know if this was related to the RGCT process itself, or rather to being asked to participate in scientific research so soon after being diagnosed with breast cancer. In any case, we cannot rule out the possibility that we underestimated the overall level of psychosocial distress due to some degree of selection bias. Given the differences observed between trial participants and nonparticipants, we need to exhibit some caution in generalizing our results to all newly diagnosed breast cancer patients who are at high risk of having hereditary breast cancer. We would note, however, that the trial participants were recruited not only from a university medical center and specialized cancer hospital but also from a number of community hospitals.

Third, although women were recruited as soon as possible after being diagnosed with breast cancer, and although the baseline questionnaire was completed by all participants before primary surgery, the time between diagnosis and completion of the baseline questionnaire varied. This could have had some influence on levels of distress. However, given the design of the trial, any effects due to the timing of the assessments would have been randomized across the two groups.

Fourth, although all women in the RGCT group underwent genetic counseling, slightly fewer than half opted for rapid DNA testing. However, we would argue that this is not so much a study limitation as it is a reflection of clinical reality. Our experience is that even at such a stressful time, patients are able to consider the options available to them and to make their own decision regarding rapid DNA testing. Previous observational studies support this position., ${ }^{4,26}$

Finally, the results of the per-protocol analyses need to be interpreted with a great deal of caution, given the limited sample size available for these analyses and the concomitant limitations in the ability to detect statistically and clinically relevant differences in psychosocial outcomes. Larger studies are needed to confirm these results.

Our study also had a number of noteworthy strengths. These include the randomized design, the multicenter setting in which it was performed, the use of standardized questionnaires, and the high data completion rates (overall, approximately 90\%).

\section{Clinical implications}

In summary, our results suggest that offering RGCT does not carry with it significant adverse psychosocial effects. However, before RGCT can be safely offered to newly diagnosed breast cancer patients who are at risk for carrying a $B R C A 1 / 2$ gene mutation, replication of these results is needed. Still, a thorough family history should be taken from all women diagnosed with breast cancer; when there is an indication for genetic counseling and testing, this should be discussed with the patient. Depending on the patients' prognosis, treatment options, and preferences, for women whose treatment decisions (may) depend on DNA test results, referral for RGCT should be considered.

\section{SUPPLEMENTARY MATERIAL}

Supplementary material is linked to the online version of the paper at http://www.nature.com/gim

\section{ACKNOWLEDGMENTS}

Funding for this study was provided by the NutsOHRA Foundation, grant SNO-T-0701-95. We thank Marianne A. Kuenen and Jacoline van der Sanden-Melis for their help with the data collection.

\section{DISCLOSURE}

The authors declare no conflict of interest.

\section{REFERENCES}

1. Rhiem K, Engel C, Graeser M, et al. The risk of contralateral breast cancer in patients from BRCA1/2 negative high risk families as compared to patients from BRCA1 or BRCA2 positive families: a retrospective cohort study. Breast Cancer Res 2012;14:R156.

2. Kiely BE, Jenkins MA, McKinley JM, et al. Contralateral risk-reducing mastectomy in BRCA1 and BRCA2 mutation carriers and other high-risk women in the Kathleen Cuningham Foundation Consortium for Research into Familial Breast Cancer (kConFab). Breast Cancer Res Treat 2010;120: 715-723.

3. Metcalfe KA, Lubinski J, Ghadirian P, et al.; Hereditary Breast Cancer Clinical Study Group. Predictors of contralateral prophylactic mastectomy in women with a BRCA1 or BRCA2 mutation: the Hereditary Breast Cancer Clinical Study Group. J Clin Oncol 2008;26:1093-1097.

4. Tercyak KP, Peshkin BN, Brogan BM, et al. Quality of life after contralateral prophylactic mastectomy in newly diagnosed high-risk breast cancer patients who underwent BRCA1/2 gene testing. J Clin Oncol 2007;25:285-291.

5. Lostumbo L, Carbine NE, Wallace J. Prophylactic mastectomy for the prevention of breast cancer. Cochrane Database Syst Rev 2010;11:CD002748.

6. Metcalfe K, Gershman S, Ghadirian P, et al. Contralateral mastectomy and survival after breast cancer in carriers of BRCA1 and BRCA2 mutations: retrospective analysis. BMJ 2014;348:g226.

7. Bresser PJ, Seynaeve C, Van Gool AR, et al. Satisfaction with prophylactic mastectomy and breast reconstruction in genetically predisposed women. Plast Reconstr Surg 2006;117:1675-1682; discussion 1683.

8. Frost MH, Slezak JM, Tran NV, et al. Satisfaction after contralateral prophylactic mastectomy: the significance of mastectomy type, reconstructive complications, and body appearance. J Clin Oncol 2005;23:7849-7856.

9. Geiger AM, West CN, Nekhlyudov L, et al. Contentment with quality of life among breast cancer survivors with and without contralateral prophylactic mastectomy. J Clin Onco/ 2006;24:1350-1356.

10. Wevers MR, Hahn DE, Verhoef $S$, et al. Breast cancer genetic counseling after diagnosis but before treatment: a pilot study on treatment consequences and psychological impact. Patient Educ Couns 2012;89:89-95.

11. Ardern-Jones A, Kenen R, Eeles R. Too much, too soon? Patients and health professionals' views concerning the impact of genetic testing at the time of 
breast cancer diagnosis in women under the age of 40. Eur J Cancer Care (Engl) 2005;14:272-281.

12. Wevers MR, Aaronson NK, Verhoef $S$, et al. Impact of rapid genetic counselling and testing on the decision to undergo immediate or delayed prophylactic mastectomy in newly diagnosed breast cancer patients: findings from a randomised controlled trial. Br J Cancer 2014;110:1081-1087.

13. Wevers MR, Ausems MG, Verhoef S, et al. Behavioral and psychosocial effects of rapid genetic counseling and testing in newly diagnosed breast cancer patients: design of a multicenter randomized clinical trial. BMC Cancer 2011;11:6.

14. The Netherlands Foundation for the Detection of Hereditary Tumours (STOET), the Dutch Society of Clinical Genetics (VKGN). Erfelijke tumoren: richtijnen voor diagnostiek en preventie. 2005.pp 10-13

15. Cohen J. Statistical Power Analysis for the Behavioral Sciences. Academic Press: New York, 1977.

16. Schou I, Ekeberg $\varnothing$, Ruland CM, Sandvik L, Kåresen R. Pessimism as a predictor of emotional morbidity one year following breast cancer surgery. Psychooncology 2004;13:309-320.

17. Hegel MT, Moore CP, Collins ED, et al. Distress, psychiatric syndromes, and impairment of function in women with newly diagnosed breast cancer. Cancer 2006;107:2924-2931.

18. Schou I, Ekeberg $\varnothing$, Sandvik L, Hjermstad MJ, Ruland CM. Multiple predictors of health-related quality of life in early stage breast cancer. Data from a year follow-up study compared with the general population. Qual Life Res 2005;14:1813-1823.

19. Schlich-Bakker KJ, Ausems MG, Schipper M, Ten Kroode HF, WárlámRodenhuis CC, van den Bout J. BRCA1/2 mutation testing in breast cancer patients: a prospective study of the long-term psychological impact of approach during adjuvant radiotherapy. Breast Cancer Res Treat 2008;109:507-514

20. Van Riel E, Wárlám-Rodenhuis CC, Verhoef S, Rutgers EJ, Ausems MG. BRCA testing of breast cancer patients: medical specialists' referral patterns, knowledge and attitudes to genetic testing. Eur J Cancer Care (Engl) 2010;19:369-376.

21. National Collaborating Centre for Cancer. National Institute for Health and Care Excellence (NICE) Guideline Familial Breast Cancer 2013: Classification and care of people at risk of familial breast cancer and management of breast cancer and related risks in people with a family history of breast cancer, 2013, https:// beta.nice.org.uk/resource/CG164/pdf/c/cg164-familial-breast-cancer-clinicalevidence-review?id=6kirgc7xvo7mh43kv7uchja5um. Accessed 5 May 2014.

22. Breast Cancer Guideline 2.0, 2013. http://www.oncoline.nl/mammacarcinoom. Accessed 7 February 2013.

23. Zilliacus E, Meiser B, Gleeson M, et al. Are we being overly cautious? A qualitative inquiry into the experiences and perceptions of treatment-focused germline BRCA genetic testing amongst women recently diagnosed with breast cancer. Support Care Cancer 2012;20:2949-2958.
24. Christie J, Quinn GP, Malo T, et al. Cognitive and psychological impact of BRCA genetic counseling in before and after definitive surgery breast cancer patients. Ann Surg Oncol 2012;19:4003-4011.

25. Hilgart JS, Coles B, Iredale R. Cancer genetic risk assessment for individuals at risk of familial breast cancer. Cochrane Database Syst Rev 2012;2:CD003721.

26. Nordin K, Roshanai A, Bjorvatn C, et al. Is genetic counseling a stressful event? Acta Oncol 2011;50:1089-1097.

27. Douma KF, Aaronson NK, Vasen HF, et al. Psychological distress and use of psychosocial support in familial adenomatous polyposis. Psychooncology 2010;19:289-298.

28. Watson $M$, Duvivier $V$, Wade Walsh $M$, et al. Family history of breast cancer: what do women understand and recall about their genetic risk? J Med Genet 1998;35:731-738.

29. Lerman C, Daly M, Masny A, Balshem A. Attitudes about genetic testing for breast-ovarian cancer susceptibility. J Clin Oncol 1994;12:843-850.

30. Horowitz M, Wilner N, Alvarez W. Impact of Event Scale: a measure of subjective stress. Psychosom Med 1979;41:209-218.

31. Brom D, Kleber RJ. De Schok Verwerkingslijst [The Impact of Event Scale]. Ned Tijdschr Psych 1985;41:164-168.

32. Aaronson NK, Ahmedzai S, Bergman B, et al. The European Organization for Research and Treatment of Cancer QLQ-C30: a quality-of-life instrument for use in international clinical trials in oncology. J Nat/ Cancer Inst 1993;85: 365-376.

33. Sprangers MA, Groenvold M, Arraras Jl, et al. The European Organization for Research and Treatment of Cancer breast cancer-specific quality-of-life questionnaire module: first results from a three-country field study. J Clin Oncol 1996;14:2756-2768.

34. Holmes-Rovner M, Kroll J, Schmitt N, et al. Patient satisfaction with health care decisions: the satisfaction with decision scale. Med Decis Making 1996;16: 58-64.

35. Zigmond AS, Snaith RP. The hospital anxiety and depression scale. Acta Psychiatr Scand 1983;67:361-370.

36. de Bock GH, Bonnema J, Zwaan RE, van de Velde CJ, Kievit J, Stiggelbout AM. Patient's needs and preferences in routine follow-up after treatment for breast cancer. Br J Cancer 2004;90:1144-1150.

37. Turner J, Kelly B, Swanson C, Allison R, Wetzig N. Psychosocial impact of newly diagnosed advanced breast cancer. Psychooncology 2005;14:396-407.

38. Spinhoven P, Ormel J, Sloekers PP, Kempen GI, Speckens AE, Van Hemert AM. A validation study of the Hospital Anxiety and Depression Scale (HADS) in different groups of Dutch subjects. Psychol Med 1997;27:363-370.

39. Pieterse AH, Ausems MG, Van Dulmen AM, Beemer FA, Bensing JM. Initial cancer genetic counseling consultation: change in counselees' cognitions and anxiety, and association with addressing their needs and preferences. Am J Med Genet A 2005;137:27-35. 